\title{
Comportamentos autolesivos, ajuste psicológico e relações familiares em adolescentes da região amazônica no Brasil
}

\author{
Maria Letícia Coelho de Oliveira \\ Carolina Lisboa \\ Pontifícia Universidade Católica do Rio Grande do \\ Sul, Brasil \\ Gina Tomé \\ Marta Reis \\ Margarida Gaspar de Matos \\ Universidade de Lisboa, Portugal
}

\author{
Diego Gómez-Baya \\ Universidad de Huelva, España
}

\author{
Juliana Maltoni \\ Carmem Beatriz Neufeld \\ Universidade de São Paulo, Brasil
}

\section{Resumo}

A adolescência é um período de importantes transformações mentais e orgânicas e uma das estratégias para a experiência de emoções negativas é a autolesão. O objetivo deste trabalho foi investigar a presença de comportamentos de autolesão em adolescentes e a associação entre a sintomatologia de ansiedade, depressão e a percepção dos adolescentes quanto ao seu relacionamento com seus pais. Este trabalho insere-se em um projeto maior, denominado Health Behavior in School-Aged Children (HBSC). O recorte se propõe investigar os adolescentes da Região Norte do Brasil (Amazônia). Os instrumentos utilizados foram o Children's Depression Inventory (CDI), o Spence Children's Anxiety Scale (SCAS) e o Questionário HBSC. A amostra foi composta de 507 estudantes de Rondônia, de 13 a 15 anos. A análise dos dados da pesquisa foi realizada por meio de medidas de comparação de média, de porcentagem (teste t e qui-quadrado) e de análise de variâncias. Os resultados apontam que os jovens que se autolesionam apresentam maiores índices de depressão e ansiedade e que a falta de percepção de apoio familiar correlaciona-se diretamente à prática da autolesão. Uma melhor compreensão da saúde mental e emocional na adolescência, proporcionará aos adolescentes maior acolhimento relacionado aos seus conflitos internos.

PalavRAs-chave

Autolesão; depressão reativa; relações familiares.

\begin{abstract}
Adolescence is a period of important mental and physical transformations, and one strategy when experiencing negative emotions is to self-harm. The aim of this study was to investigate the presence of self-injurious behaviors in adolescents, and their association between anxiety symptomatology, depression and adolescents' perception of their relationship with parents. This work is part of a larger project called Health Behavior in School-Aged Children (HBSC). Proposed scope is to investigate adolescents of the Northern Region of Brazil (Amazon). Instruments used were Children Depression Inventory (CDI), Children Spence Anxiety Scale (SCAS), and HBSC Questionnaire. Sample consisted of 507 students from Rondônia, aged 13 to 15 years. Data analysis was performed through comparison of means and percentages (t-test and chi-square) and analysis of variance. Results indicate that young people who self-harm have higher rates of depression and anxiety and that lacking perception of family support correlates directly with self-harm behavior. A better understanding of mental and emotional health in adolescence will provide adolescents with greater support for their internal conflicts.
\end{abstract}

KEYWORDS

Self-harm; reactive depression; family relationships. 
Comportamentos autolesivos, ajuste psicológico e relações familiares em adolescentes da região amazônica no Brasil

Acredita-se que os sujeitos que se lesionam fariam isso por não saberem lidar com emoções fortes, pressões externas e problemas de relacionamento. Essas ações seriam uma maneira de administrarem sentimentos pela via da atuação, em vez de expressá-las verbalmente, pois o outro a ser destruído, pelo ato agressivo, estaria internalizado. Assim, lesionar-se seria uma forma de amenizar a angústia, gerando simultaneamente dor e prazer (Cedaro \& Nascimento, 2013). Lembranças estressantes, ansiedade ou depressão poderiam atuar como gatilho para ativar esse mecanismo disfuncional de regulação das emoções e ajudar a consolidar a autolesão como um padrão de resposta (Arcoverde \& Soares, 2011).

Todavia, cabe aos profissionais da psicologia e a todos aqueles que convivem com pessoas na chamada fase vital da adolescência, despojar-se da visão preconceituosa incrustrada de que se trata de um período conturbado, crítico e violento (Cerqueira-Santos, Neto, \& Koller, 2014), para que a família ajude o jovem, começando pela reconstrução da sua autoestima, restabelecendo os laços afetivos que muitas vezes, até por distração, podem ter sido negligenciados e por isso mesmo ter proporcionado o isolamento e sentimento de abandono do adolescente. É fundamental para a cura, não só o acompanhamento psicológico, mas também atividades familiares de lazer, atividades lúdicas e desportivas que sejam do agrado do jovem e que lhe permitam desenvolver as suas competências e extinguir a violência au- todirigida da sua vida (Reis, Figueira, Ramiro, \& Matos, 2012).

A ansiedade relaciona-se ao desespero e à desesperança, gerando uma expectativa de sofrimento constante (Darwin, 2009). Um dos componentes cognitivos mais importantes da ansiedade é a preocupação, e na adolescência, a natureza dos temores está mais baseadaem comparações sociais,sendo comum a ansiedade em relação à falhas, críticas e aparência física (Warren \& Sroufe, 2004).

Pessoas com ansiedade também podem sofrer de depressão, o que torna debilitante a capacidade de sentir prazer, prejudica a interação e a participação de uma vida ativa, acarretando em efeitos nocivos à saúde, e a durabilidade pode ser de meses ou anos (Barlow, 2016). Sujeitos deprimidos distorcem suas interpretações de eventos, mantendo visões negativas sobre si, o ambiente e sobre o futuro. A relativa inatividade contribui para o humor depressivo, pois, assim, o indivíduo carece de oportunidades para obter um sentimento de domínio, o que o conduz para mais pensamentos negativos (Beck, 2013). Promover a capacidade pessoal para lidar positivamente com problemas poderia evitar a depressão, uma vez que o aumento da depressão pode estar atrelado ao modo como os indivíduos, incluindo crianças e adolescentes, lidam com o conflito (Tomé \& Matos, 2006).

As famílias, os meios de comunicação e a escola fazem parte de um contexto importante para a educação e a promoção de saúde e têm uma relação direta com os contextos sociais em que estão inseridos (Neufeld, 2015). Durante a adolescência, há evidências de que 
o processo de aquisição da autonomia em relação aos pais, assim como as demais tarefas desenvolvimentais da fase, são influenciados por características como o estilo educativo destes cuidadores (Benchaya, Bisch, Moreira, Ferigolo, \& Barros, 2011). Estudar e pensar sobre riscos em interação com fatores protetivos pode ser a chave ou um caminho eficaz para o sucesso de ações focais e preventivas que visem à saúde dos jovens (Lisboa, Wendt, Neufeld, \& Matos, 2014).

Este projeto teve por finalidade oferecer uma contribuição empírica para a compreensão da associação entre sintomas de depressão, ansiedade e a percepção dos adolescentes no que se refere ao seu relacionamento com seus pais. Pode-se dizer que, em geral, é no interior da família que o indivíduo mantém seus primeiros relacionamentos interpessoais com pessoas significativas, estabelecendo trocas emocionais que funcionam como um suporte afetivo importante quando os indivíduos atingem a idade adulta. Enfatiza-se a importância de mais estudos descritivos que visem o levantamento de fatores de risco e proteção à saúde de adolescentes. Foi ainda objetivo verificar as diferenças e semelhanças entre meninos e meninas do interior e da capital de Rondônia, região escolhida para representar a Região Norte do Brasil (Região Amazônica), tomando por referência uma amostra de participantes de 13 a 15 anos, estudantes de escola públicas. No que toca a Rondônia, este Estado, antes Território Federal (até 1982) e a Amazônia, em geral, eram apresentadas como vazio demográfico, desconsiderando-se, portanto, os povos que habitavam, como indígenas, quilombolas e caboclos radicados nas margens dos rios há várias gerações. O novo processo de colonização iniciado na década de 1970, sob a coordenação do Estado brasileiro, recortou o território de Rondônia e fez surgir, 52 municípios, dos quais a maioria tem menos de 40 mil habitantes e sobrevive, majoritariamente, da agricultura, da pecuária, do comércio e do funcionalismo público (Barbosa, 2015). As hipóteses desta pesquisa foram de que os adolescentes que disseram já ter praticado a autolesão teriam um índice maior de ansiedade e depressão, que a prática seria predominante nas meninas e na capital do Estado de Rondônia, e de que os adolescentes com maior índice de percepção de apoio familiar teriam menor índice de autolesão.

\section{Método}

O presente trabalho insere-se em um projeto maior, denominado Health Behavior in School-Aged Children (HBSC), que é um extenso estudo internacional (www.hbsc.org) (Currie et al., 2004, 2012) que tem como objetivo mapear e comparar os indicadores e comportamentos de saúde e bem-estar de adolescentes, que em colaboração com a Organização Mundial da Saúde, diz respeito a um estudo transnacional e vem sendo conduzido em 43 países da Europa e América do Norte, há mais de 30 anos. O Brasil foi incluído para atuar no International Linked Project no estudo HBSC/OMS em 2016 (Nogueira, 2017) e desde então o estudo que vem sendo realizado em 6 Estados do país sob coordenação do LaPICC-USP.

\section{Participantes}

Participaram do estudo 507 adolescentes de Rondônia, de 13 a 15 anos, representando 
a Região Norte do Brasil com média de idade de $13,9(D P=1,03)$, sendo que $61,3 \%$ do total eram meninas. Os participantes foram selecionados de modo aleatório em 8 escolas de Rolim de Moura, no interior de Rondônia, representando 54,5\% e 9 escolas na capital do Estado, em Porto Velho.

\section{Instrumentos}

Os instrumentos utilizados foram o Children Depression Inventory (CDI), que visa avaliar a depressão juvenil (Kovacs, 1983), traduzido e adaptado por Gouveia, Barbosa, Almeida, e Gaião (1995), o Spence Children Anxiety Scale (SCAS) para avaliar a ansiedade nos adolescentes (Spense, 1998), em sua versão adaptada para o português (Escala Spense de Ansiedade Infantil) por DeSouza et al. (2014), e questões referentes à percepção de apoio familiar e à prática de autolesão do Questionário do Health Behaviour in School-aged Children na sua versão portuguesa (HBSC), que procura obter dados do tipo epidemiológico, e são usados em Portugal desde 1998, e na Europa, desde 1983, de 4 em 4 anos, tendo sido adapatado para a população brasileira em 2014 por Nogueira (2017). Estudos sobre a validade e as propriedades psicométricas dos itens incluídos já foram publicados (Roberts et al., 2009).

No que se refere à autolesão, a questão utilizada teve como questionamento se "Nos últimos 12 meses, você já se feriu de propósito, como se cortar, por exemplo?" e as alternativas de respostas: Sim ou Não. Referente à percepção do relacionamento familiar, foram avaliadas 3 categorias de questões. A primeira categoria apresentou a seguinte questão:
"O quanto se sente a vontade para falar sobre os assuntos que mais te preocupam com as seguintes pessoas?" e foram avaliadas as respostas em relação ao Pai e à Mãe, e as categorias de respostas foram: "Muito fácil, Fácil, Difícil e Muito difícil". A segunda questão referente ao relacionamento familiar apresentava uma escala likert de 1 a 7 para ser assinalada quanto à Discordar muito fortemente ou Concordar muito fortemente, e as questões foram: "A minha família realmente tenta me ajudar", "Eu tenho o apoio emocional que preciso da minha família", "Eu posso falar com a minha família sobre os meus problemas" e "A minha família está disponível para me ajudar a tomar decisões". E por fim, a última questão avaliativa do relacionamento familiar trazia a seguinte pergunta: "O quanto você acha que seus pais ou responsáveis sabem sobre":"Quem são seus amigos," "Onde você gasta o seu dinheiro" e" O que você costuma fazer em seu tempo livre", e as categorias de respostas foram: Muito, Mais ou menos, Pouco e Nada.

\section{Procedimentos}

Após o treinamento para a realização da coleta de dados, junto à equipe da coordenação do projeto HBSC no Brasil, em Ribeirão Preto, cidade em que se localiza o LaPICC, Laboratório de Pesquisa e Intervenção Cognitivo-Comportamental da Universidade de São Paulo, foi contatada a Secretaria Estadual de Educação tanto no interior quanto na capital de Rondônia e em seguida ao levantamento das escolas públicas, realizou-se uma seleção aleatória das instituições de ensino a serem convidadas, 
de acordo com a amostragem e respeitando a proporção entre as turmas de cada região.

Após o convite às escolas e seu aceite, realizou-se a seleção por conveniência das turmas a serem convidadas para participarem do estudo. Na sequência foi feito o convite aos alunos nas salas de aula referente às idades selecionadas e entregue os Termos de Consentimento Informado, Livre e Esclarecido (TCLE) e o de Consentimento para a Formação de Banco de Dados para que os pais/responsáveis daqueles que se interessaram em participar assinassem e a participação do estudante ficou condicionada em primeiro lugar ao aceite da escola, em seguida à autorização formal dos pais e, por fim, ao assentimento dos adolescentes atestado no dia previamente combinado com a direção escolar para a realização da pesquisa.

Para a coleta de dados foi selecionada uma equipe composta por psicólogos e estudantes de psicologia. A pesquisa foi conduzida pela equipe com aplicações coletivas em salas de aula ou outros locais oferecidos pelas escolas (laboratório de informática, sala de leitura e refeitório). Os participantes foram informados que poderiam sair da pesquisa a qualquer momento sem que ocorresse nenhuma punição ou dano. Se fossem identificadas demandas ou mal-estar/sofrimento, seriam realizados encaminhamentos dos participantes através da identificação do cabeçalho da escala SCAS para o Serviço de Clínica Escola da Faculdade de Psicologia da respectiva cidade, tanto no que se refere ao interior quanto à capital do Estado de Rondônia. Os procedimentos metodológicos mais aprofundados do estudo estão descritos em publicações anteriores em níveis nacional e internacional (Matos et al., 2011; Currie et al., 2004, 2012; Roberts et al., 2009).

O projeto seguiu as diretrizes e normas do Conselho Nacional da Saúde, regulamentadoras da Resolução no 466/2012. Foram respeitados todos os princípios éticos para pesquisa com seres humanos. Todos os participantes tiveram sua identidade preservada, souberam do risco mínimo gerado por possível emoção desconfortável devido a perguntas pessoais e os possíveis encaminhamentos, se fossem necessários. $O$ estudo seguiu todas as normas de aprovação científica, ética e administrativa e teve o consentimento informado de todos os diretores das escolas, adolescentes participantes e respectivos encarregados de educação.

\section{Análise dos dados}

Os dados foram analisados no programa estatístico SPSS versão 24. Foi realizada estatística descritiva, qui quadrado, análise de variâncias e diferenças de médias (t teste) e porcentagem para as variáveis analisadas.

\section{Resultados}

A Tabela 1 apresenta a estatística comparativa por sexo e, regiões, não se observando diferenças estatisticamente significativas.

A Tabela 2 demostra a diferença entre a prática de autolesões para a sintomatologia da ansiedade e depressão. Os resultados apontam que os jovens que se autolesionam apresentam maiores índices de depressão $(M=16,7 ; D P=10,4)$ e maiores índices de ansiedade $(M=45,8 ; D P=16,7)$.

A Tabela 3 e a Tabela 4 apresentam as diferenças entre as estatísticas descritivas e comparativas sobre a prática de autolesão dos 
Tabela 1

Diferenças entre Prática de Autolesões para sexo e região

\begin{tabular}{|c|c|c|c|c|c|c|c|c|}
\hline \multicolumn{9}{|c|}{ Prática de Autolesão } \\
\hline & & \multicolumn{2}{|c|}{ Sim } & \multicolumn{2}{|c|}{ Não } & \multirow[t]{2}{*}{ Total } & \multirow[t]{2}{*}{$X^{2}$} & \multirow[t]{2}{*}{ gl } \\
\hline & & $\mathrm{N}$ & $\%$ & $\mathrm{~N}$ & $\%$ & & & \\
\hline \multirow{2}{*}{ Sexo } & Meninos & 38 & 19,4 & 158 & 80,6 & 196 & 1,978 & 1 \\
\hline & Meninas & 77 & 24,8 & 234 & 75,2 & 311 & & \\
\hline \multirow{2}{*}{ Região } & $\begin{array}{l}\text { Porto } \\
\text { Velho }\end{array}$ & 71 & 25,5 & 207 & 74,5 & 278 & 3,129 & 1 \\
\hline & $\begin{array}{l}\text { Rolim de } \\
\text { Moura }\end{array}$ & 44 & 19 & 188 & 81 & 232 & & \\
\hline
\end{tabular}

Tabela 2

Comparação de médias entre prática de autolesão para depressão e ansiedade

\begin{tabular}{ccccccccc}
\hline $\begin{array}{l}\text { Prática de } \\
\text { Autolesão }\end{array}$ & Sim & Não & \multicolumn{10}{c}{ (10 } & & & & \\
\hline & $N$ & $M$ & $D P$ & $N$ & $M$ & $D P$ & $t$ & $p$ \\
Depressão & 115 & 16,7 & 10,4 & 395 & 9,2 & 7,5 & 8,692 & .000 \\
Ansiedade & 115 & 45,8 & 16,7 & 395 & 34,2 & 15,9 & 6,779 & .000 \\
\hline
\end{tabular}

adolescentes e a percepção da relação familiar, com as variáveis de dificuldade em conversar com o pai e com a mãe (categorias de respostas variando entre Muita dificuldade e Muita facilidade). O suporte foi avaliado por uma escala de 4 itens, contendo afirmativas sobre ser ajudado, apoio emocional, falar sobre problemas e ajuda na tomada de decisões. As respostas de ambas as escalas possuem cinco categorias de respostas variando entre Discordo fortemente a Concordo fortemente. A supervisão parental foi avaliada através das questões do quanto os pais sabem sobre quem são seus amigos; onde gastam o dinheiro e o que os filhos costumam fazer em seu tempo livre, e as categorias de respostas foram: Muito, Mais ou menos, Pouco e Nada.

Para as variáveis conversar com a mãe e com o pai, os dados não foram estatisticamente sig- nificativos. Os jovens sem prática de autolesão consideram que os seus pais sabem muito sobre quem são os seus amigos (47,6\%; X2 = 16,052; gl.= $4, p<.01)$, que os seus pais sabem muito sobre onde gastam o seu dinheiro $\left(49,1 \% ; X^{2}\right.$ $=16,784 ; g l .=3, p<.001)$ e que os seus pais sabem muito sobre o que costuma fazer no seu tempo livre (54,8\%; $x^{2}=21,543 ;$ gl. $\left.=4 ; p<.001\right)$, quando comparados com os que têm prática de autolesões.

Em relação ao suporte familiar, os jovens que não têm prática de autolesão tem média superior de percepção de ajuda familiar $(M=$ 5,3; $D P=2,1$ ), de poder falar dos problemas com a família $(M=4,7 ; D P=2,4)$ e de ajuda a tomar decisões $(M=5,4 ; D P=2,2)$. Para a percepção de apoio emocional os resultados não foram estatisticamente significativos. 
Tabela 3

Diferenças entre prática de Autolesão e relacionamento Familiar

Dificuldade em conversar com o pai

\begin{tabular}{|c|c|c|c|c|c|c|c|c|c|c|c|c|c|c|}
\hline & & $\begin{array}{l}\text { Muito } \\
\text { Fácil }\end{array}$ & Fácil & Difícil & $\begin{array}{l}\text { Muito } \\
\text { Difícil }\end{array}$ & $\begin{array}{c}\text { Não } \\
\text { tenho } \\
\text { ou não } \\
\text { vejo }\end{array}$ & Total & $x^{2}$ & $\mathrm{Gl}$ & & & & & \\
\hline & & $\mathrm{N}$ & $\%$ & $\mathrm{~N}$ & $\%$ & $\mathrm{~N}$ & $\%$ & $\mathrm{~N}$ & $\%$ & $\mathrm{~N}$ & $\%$ & & & \\
\hline \multirow[t]{2}{*}{$\begin{array}{l}\text { Prática de } \\
\text { Autolesão }\end{array}$} & Sim & 17 & 15,5 & 20 & 18,2 & 20 & 18,2 & 31 & 28,2 & 22 & 20 & 110 & 5,311 & 5 \\
\hline & Não & 76 & 20,4 & 78 & 21 & 78 & 21 & 72 & 19,4 & 67 & 18 & 371 & & \\
\hline \multicolumn{15}{|c|}{ Dificuldade em conversar com a mãe } \\
\hline \multirow{4}{*}{$\begin{array}{l}\text { Prática de } \\
\text { Autolesão }\end{array}$} & & $\begin{array}{l}\text { Muito } \\
\text { Fácil }\end{array}$ & Fácil & Difícil & $\begin{array}{l}\text { Muito } \\
\text { Difícil }\end{array}$ & $\begin{array}{c}\text { Não } \\
\text { tenho } \\
\text { ou não } \\
\text { vejo }\end{array}$ & Total & $x^{2}$ & Gl & & & & & \\
\hline & & $\mathrm{N}$ & $\%$ & $\mathrm{~N}$ & $\%$ & $\mathrm{~N}$ & $\%$ & $\mathrm{~N}$ & $\%$ & $\mathrm{~N}$ & $\%$ & & & \\
\hline & Sim & 33 & 30,6 & 25 & 23,1 & 24 & 22,2 & 19 & 17,6 & 6 & 5,6 & 107 & 5,311 & 5 \\
\hline & Não & 147 & 40,5 & 111 & 30,6 & 62 & 17,1 & 34 & 9,4 & 9 & 2,5 & 363 & & \\
\hline \multicolumn{15}{|c|}{ Quanto os seus pais/responsáveis sabem sobre quem são seus amigos } \\
\hline \multirow{4}{*}{$\begin{array}{l}\text { Prática de } \\
\text { Autolesão }\end{array}$} & & Muito & $\begin{array}{c}\text { Mais ou } \\
\text { menos }\end{array}$ & Pouco & Nada & Total & $x^{2}$ & Gl & & & & & & \\
\hline & & $\mathrm{N}$ & $\%$ & $\mathrm{~N}$ & $\%$ & $\mathrm{~N}$ & $\%$ & $\mathrm{~N}$ & $\%$ & & & & & \\
\hline & Sim & 37 & 32,2 & 44 & 38,3 & 20 & 17,4 & 14 & 12,2 & 115 & $16,052^{* *}$ & 4 & & \\
\hline & Não & 187 & 47,6 & 127 & 32,3 & 62 & 15,8 & 16 & 4,1 & 392 & & & & \\
\hline \multicolumn{15}{|c|}{ Quanto seus pais/ responsáveis sabem sobre onde você gasta seu dinheiro } \\
\hline \multirow{4}{*}{$\begin{array}{l}\text { Prática de } \\
\text { Autolesão }\end{array}$} & & Muito & $\begin{array}{c}\text { Mais ou } \\
\text { menos }\end{array}$ & Pouco & Nada & Total & $x^{2}$ & $\mathrm{Gl}$ & & & & & & \\
\hline & & $\mathrm{N}$ & $\%$ & $\mathrm{~N}$ & $\%$ & $\mathrm{~N}$ & $\%$ & $\mathrm{~N}$ & $\%$ & & & & & \\
\hline & Sim & 38 & 33,9 & 27 & 24,1 & 28 & 25 & 19 & 17 & 112 & $16,784^{* * *}$ & 3 & & \\
\hline & Não & 188 & 49,1 & 108 & 28,2 & 53 & 13,8 & 34 & 8,9 & 383 & & & & \\
\hline \multicolumn{15}{|c|}{ Quanto seus pais/ responsáveis sabem sobre o que costuma fazer no seu tempo livre } \\
\hline \multirow{4}{*}{$\begin{array}{l}\text { Prática de } \\
\text { Autolesão }\end{array}$} & & Muito & $\begin{array}{c}\text { Mais ou } \\
\text { menos }\end{array}$ & Pouco & Nada & Total & $x^{2}$ & $\mathrm{Gl}$ & & & & & & \\
\hline & & $\mathrm{N}$ & $\%$ & $\mathrm{~N}$ & $\%$ & $\mathrm{~N}$ & $\%$ & $\mathrm{~N}$ & $\%$ & & & & & \\
\hline & Sim & 36 & 31,9 & 29 & 25,7 & 17 & 15 & 30 & 26,5 & 112 & $21,543^{* * *}$ & 4 & & \\
\hline & Não & 212 & 54,8 & 82 & 21,2 & 39 & 10,1 & 53 & 13,7 & 386 & & & & \\
\hline
\end{tabular}

\section{Discussão}

Dados internacionais revelam que cerca de 10\% dos adolescentes já terão tido pelo menos um episódio de autolesão ao longo da sua vida.
A investigação, a identificação e a prevenção destes comportamentos são consideradas prioritárias nas políticas de saúde da União Europeia (Guerreiro, Sampaio, \& Figueira, 2014). 
Tabela 4

Comparação de médias entre prática de autolesão e relacionamento familiar

\begin{tabular}{|c|c|c|c|c|c|c|c|c|}
\hline Prática de Autolesão & Sim & & Não & & & & & \\
\hline & $N$ & $M$ & $D P$ & $N$ & $M$ & $D P$ & $t$ & $p$ \\
\hline Sente que a família tenta ajudar & 113 & 4,6 & 2,4 & 381 & 5,3 & 2,1 & $-2,832$ & .005 \\
\hline $\begin{array}{l}\text { Sente que a família apoia } \\
\text { emocionalmente }\end{array}$ & 112 & 4,8 & 2,6 & 376 & 5 & 2,3 & -.970 & .333 \\
\hline $\begin{array}{l}\text { Sente que pode falar dos problemas } \\
\text { com a família }\end{array}$ & 111 & 4 & 2,7 & 371 & 4,7 & 2,4 & $-2,433$ & .015 \\
\hline $\begin{array}{l}\text { Sente que a família está disposta a } \\
\text { ajudar a tomar decisões }\end{array}$ & 112 & 4,8 & 2,5 & 379 & 5,4 & 2,2 & $-2,263$ & .024 \\
\hline
\end{tabular}

Em Portugal, o estudo nacional (Health Behaviour in School-aged Children) realizado em 2010, com uma amostra de 5050 adolescentes, com um média de 14 anos de idade, verificouse que $15,6 \%$ dos adolescentes mencionaram ter-se autoagredido pelo menos uma vez nos últimos 12 meses (Matos et al., 2011). De acordo com esse mesmo estudo, o sentimento mais comum no momento do ato foi estar triste $(18,5 \%)$, nervoso $(12,5 \%)$ e bravo $(9,1 \%)$, o que demonstra os diferentes construtos que avaliam o comportamento (Matos et al., 2015). Os comportamentos de autolesão na amostra do Estado de Rondônia ocorreram em 23\% dos adolescentes, comparado aos resultados da pesquisa do HBSC de Portugal, de acordo com o relatório de 2011, houve um acréscimo de $7,4 \%$ no percentual, no entanto, a última versão portuguesa relata que $42,2 \%$ dos jovens afirmaram ter tido lesões nos últimos 12 meses (Matos et al., 2018), o que faz com que o índice passe a ser $19,2 \%$ menor do que os recentes dados da ocorrência das autolesões nos adolescentes portugueses, sendo que os dados de Rondônia, quando comparados à amostra da pesquisa do HBSC realizada no interior do Estado de São Paulo, com adolescentes de 13 anos, houve uma redução no percentual de 5,5\% menor incidência, sendo ambas as amostras de análises de autolesão mais frequentes nas meninas (Nogueira, 2017). No entanto, a literatura diverge quanto a associação com o sexo, sendo que Matos et al. (2015) encontraram associações positivas com o sexo feminino, enquanto Barrocas et al. (2015) com o sexo masculino, diferença a qual não representou significância quanto aos dados rondonienses. As relações de gênero, de separações históricas entre meninos e meninas e que ainda hoje causam polêmicas dentro das escolas que sofre alguma forma de opressão é a naturalização de determinadas marcas - estigmas - que deixam profundas cicatrizes sociais, causando transtornos na saúde mental - emocional/sentimental - do indivíduo afetado, e por essa razão precisamos debater diuturnamente as relações sociais apontadas para que consigamos naturalizar, entre todos e todas, o respeito, a dignidade e a tolerância humana (Silva, 2013).

Os jovens que se auto lesionam apresentam maior tendência para uma sintomatologia para depressão e ansiedade, o que corrobora as pesquisas feitas por Guerreiro et al. (2014), nas quais jovens que relatavam autolesão 
apresentavam maior sintomatologia depressiva e ansiosa, assim como maiores taxas de consumo de álcool, de embriaguez, de consumo de tabaco e de utilização de drogas ilegais (Guerreiro et al., 2014). Estes dados confirmam que os transtornos de ansiedade se constituem em uma das condições mais frequentes e deteriorantes dentre os transtornos emocionais entre crianças e adolescentes, estimandose que $11 \%$ dos adolescentes sofram de transtorno de ansiedade (Costello, Egger, Copeland, \& Angold, 2011).

Em função das modificações nos níveis cognitivo e afetivo durante a adolescência, a atenção a fatores como autoestima e capacidade de regulação emocional dos adolescentes também pode ser importante para profissionais comprometidos com a promoção da saúde. Dessa forma, compreende-se a importância de trabalhar os componentes da autoestima e da expressão/supressão de emoções para a promoção de contextos que estimulem a aquisição das habilidades exigidas para a consecução das tarefas desenvolvimentais da adolescência (Macedo, Petersen, \& Koller, 2017).

Estudos apontam que o tipo de vínculo estabelecido entre pais e filhos desde os primeiros anos de vida é um importante fator para a promoção de saúde mental em todas as faixas etárias (Jordão \& Ramires, 2010). Os achados da amostra do HBSC com os adolescentes da região Sudeste acima citada, refere-se que o suporte familiar percebido e a percepção acerca da qualidade de comunicação familiar apresentam-se inferiores aos dados portugueses (Matos et al., 2015), mas ainda podem ser considerados positivos para a maioria dos adolescentes em questão. São os meninos que percebem mais o suporte familiar e atualidade da comunicação com os membros da família, convergindo com os resultados internacionais de Inchley et al. (2016) e Matos et al. (2015), respectivamente.

Comparando os resultados das pesquisas do HBSC do Estado de Rondônia com as HBSC do Estado de São Paulo, contatou-se que da mesma forma a comunicação com os membros da família apresenta-se frequentemente mais fácil com as mães do que com os pais. No entanto, na amostra de Rondônia não pareceu ser significativo quando correlacionado essa dificuldade de conversa com o pai à presença de comportamentos autolesivos, o que ao contrário, em relação a ter dificuldade em conversar com a mãe, os dados constataram que houve uma considerável significância. Nota-se também que a falta de percepção de apoio familiar correlaciona-se diretamente à prática da autolesão. Isto demonstra que investimentos devem ser feitos na relação com os pais, para incluí-los como uma fonte maior de apoio.

Há estudos mostrando que uma adequada e consistente relação com os pais pode funcionar como fator de proteção em relação ao desenvolvimento de psicopatologias na infância e na adolescência, enquanto uma relação insegura tende a deixar os adolescentes em situação de vulnerabilidade emocional e afetiva (Tortorelli, Carreiro \& Araújo, 2010). Para que isso ocorra, é indispensável que as ações consigam integrar os pais/responsáveis e demais familiares, em especial as figuras maternas. (Nogueira, 2017). Por sua vez, tal vulne- 
rabilidade pode acarretar o envolvimento em situações de risco (Benchaya et al., 2011; Ok \& Aslan, 2010; Tortorelli et al., 2010), indicando uma possível relação entre os fenômenos em estudo, direcionando uma compreensão mais ampla e integradora dessas inter-relações.

O presente estudo tem alguns aspectos a serem valorizados. Primeiro, a dimensão e a representatividade da amostra, também por ter sido em uma região em desenvolvimento com características tão específicas no Brasil, e pelos resultados da estatística inferencial poderem ser comparados para o contexto nacional onde o estudo foi realizado e também internacional, do qual é representativo, com uma margem de erro reduzida. Além disso, como limitações, pode-se referir a transversalidade dos dados, que não permite estabelecer uma relação de causalidade entre as variáveis analisadas e, também, o fato desta pesquisa se basear em medidas de autopercepção.

No entanto, a investigação tem o aspecto positivo de se tratar de um estudo nacional com amostra representativa, como já referido, e, ainda, estar integrada a um projeto maior, com colaboração internacional. Mesmo com limitações, salienta-se a importância desta pesquisa na medida em que se investigaram fatores de risco e de proteção em adolescentes.

As hipóteses desta pesquisa foram confirmadas, demonstrando que as meninas foram predominantes entre os adolescentes que já praticaram a autolesão, que a prevalência foi na capital do Estado de Rondônia e que os adolescentes que se utilizam da prática da autolesão tiveram um índice maior de ansiedade e depressão, sendo que os adolescentes com maior índi- ce de percepção de apoio familiar tiveram menor índice de autolesão, conforme o previsto pelas hipóteses. A autolesão é uma violência negativa e prejudicial, que está relacionada à formação da identidade, tarefa desenvolvimental central na adolescência. Ou seja, segundo a prevalência identificada e as relacões à sintomas ansiosos e depressivos, ela ocorre com frequência significativa na vida dos jovens, sendo necessário que os profissionais e políticas públicas reflitam sobre esse fenômeno e sobre formas de intervenção.

Enquanto não há medicamentos específicos para o tratamento da violência autodirigida, a medicação que habitualmente é usada é a direcionada para os sintomas de depressão, ansiedade e/ou impulsividade. Outros aspetos do tratamento/intervenção podem incluir o trabalho da família, as intervenções com a escola, e aumentar os níveis de apoio (Reis et al., 2012).

Estima-se a importância do desenvolvimento de habilidades para o bem-estar objetivando instrumentalizar pais e educadores a lidar com as questões emocionais das crianças e adolescentes, visando prevenir psicopatologias decorrentes da inabilidade de lidar com as emoções, como a ansiedade, violência, depressão e problemas psicossomáticos. Faz parte da educação emocional ter consciência dos próprios estados emocionais e dispor de recursos para gerir esses estados, isto é, tornar-se emocionalmente educado sendo mais consciente sobre as próprias emoções perturbadoras e sendo capaz de manter interações pessoais saudáveis (Rodrigues, 2015).

A promoção de competências pessoais e sociais e sua utilização na área de desenvolvimento social e na promoção de saúde pode constituir 
uma estratégia na construção de alternativas, na adopção de modos alternativos de lidar com os desafios da vida (Matos \& Sampaio, 2009). Nesse sentido, faz-se necessário o olhar, a discussão sobre as condições de desenvolvimento e a proposta de um modelo de intervenção, e um conjunto de atividades associadas à promoção de competências pessoais, sociais e ambientais, trabalhando com crianças e adolescentes, nesta perspectiva de os ajudar a preparar-se para a construção do seu futuro com mais e melhores competências, baseado em apoio continuado e transversal e que em suma se transforme numa cultura institucional (Matos et al., 2015).

\section{Referências}

Arcoverde, R. L., \& Soares, L. S. L. (2011). Funções Neuropsicológicas Associadas a Condutas Autolesivas: Revisão Integrativa de Literatura. Psicologia: reflexão e crítica, 25(2), 293-300. https://doi.org/10.1590/ S0102-79722012000200011

Barlow, D. H. (2016). Manual clínico dos transtornos psicológicos: tratamento passo a passo. Porto Alegre, Artmed.

Barbosa, X. C. (2015). Território e saúde: políticas públicas de combate à dengue em Porto Velho/RO, 1999-2013. Tese de Doutorado. Universidade Federal de Rondônia, Porto Velho, RO.

Barrocas, A. L., Giletta, M., Hankin, B. L., Prinstein, M. J., \& Abela, J. R. Z. (2015). Nonsuicidal selfinjury in adolescence: longitudinal course, trajectories, and intrapersonal predictors. Journal of Abnormal Child Psychology, 43, 369-380. https://doi.org/10.1007/s10802014-9895-4

Beck, J. (2013). Terapia cognitivo-comportamental: teoria e prática. Porto Alegre: Artmed.

Benchaya, M. C., Bisch, N. K., Moreira, T. C., Ferigolo, M., \& Barros, H. M.T. (2011). Pais não autoritativos e o impacto no uso de drogas: a percepção dos filhos adolescentes. Jornal de Pediatria, 87(3), 238-244. https://dx.doi. org/10.1590/S0021-75572011000300010

Cedaro, J. J., \& Nascimento, J. P. G. (2013). Dor e gozo: Relatos de mulheres jovens sobre automutilações. Psicologia USP, 24(2), 203-223. https://doi.org/10.1590/S010365642013000200002

Cerqueira-Santos, E., Melo Neto, O. C., \& Koller, S. H. (2014). Adolescentes e Adolescências. En L. F. Habgzang, E. D. B. Dei Schiro, \& S. H. Koller, Trabalhando com Adolescentes, pp. 17-29. Porto Alegre: Artmed.

Costello, E., Egger, H., Copeland, W., Erkanli, A., \& Angold, A. (2011). The developmental epidemiology of anxiety disorders: Phenomenology, prevalence, and comorbidity. In W. Silverman, \& A. Field (Eds.), Anxiety Disorders in Children and Adolescents, pp. 56-75. Cambridge: Cambridge University Press. https://doi. org/10.1017/CBO9780511994920.004

Currie, C., Roberts, C., Morgan, A., Smith, R., Settertbulte, W., Samdal, O., \& Rasmussen, V. (Eds.). (2004). HBSC and WHO cross national study: research protocol for the 2001/2002 survey. Copenhagen: World Health Organization.

Currie, C., Zanotti, C., Morgan, A., Currie, D., \& Barnekow, V. (Eds.). (2012). Social determinants of health and well-being among young people: Health Behaviour in Schoolaged Children (HBSC) study: International Report From the 2009/2010 survey. Copenhagen: World Health Organization. Recuperado de http://www. euro.who. int/_data/assets/pdf_file/0003/163857/ Social-de-terminants-of-health-and-wellbeing-among-young-people.pdf

Darwin, C. (2009). A expressão das emoções no homemenos animais. São Paulo: Companhia das letras.

DeSousa, D. A., Pereira, A. S., Petersen, C.S., Manfro, G.G., Salum, G.A., \& Koller, S.H. (2014). Psychometric Properties of the Brazilian-Portuguese version of the Spence Children's Anxiety Scale (SCAS): self- and parent-report versions. Journal of Anxiety Disorders, 28(5), 427-436. https://doi. org/10.1016/j.janxdis.2014.03.006 
Edelman, S. (2014). Basta pensar diferente: como a ciência pode ajudar você a ver o mundo por novos olhos. São Paulo: Editora Fundamento Educacional.

Gouveia, V. V., Barbosa, G. A., Almeida, H. J. F., \& Gaião, A. A. (1995). Inventário de Depressão Infantil - CDI: estudo de adaptação com escolares de João Pessoa. Jornal Brasileiro de Psiquiatria, 44, 345-349.

Guerreiro, D. F., Sampaio, D. \& Figueira, M. L. (2014). Relatório da investigação "Comportamentos autolesivos em adolescentes:Características epidemiológicas $e$ análise de fatores psicopatológicos, temperamento afetivo e estratégias de coping". Lisboa: Universidade de Lisboa.

Inchley, J., Currie, D., Young, T., Samdal, O., Torsheim, T., Augustson, L., et al. (Eds.). (2016). Growing up unequal: gender and socioeconomic differences in young people's health and well-being. Health Behaviour in School-aged Children (HBSC) study: international report from the 2013/2014 survey. Copenhagen: WHO Regional Office for Europe (Health policy for children and adolescents, No 7).

Jordão, A. B., \& Ramires, V. R. R. (2010). Vínculos Afetivos de Adolescentes Borderline e seus Pais. Psicologia: Teoria e Pesquisa, 26(1), 89-98. http://dx.doi.org/10.1590/S010237722010000100011

Kovacs, M. (2003). Children's Depression Inventory (CDI): Technical Manual Update. Toronto: Multhi-Health Systems Inc.

Lisboa, C. S. M., Wendt, G. W., Neufeld, C. B., \& Matos, M. G. (2014). Satisfação com a vida e com a família e violência interpessoal na adolescência. Revista Brasileira de Terapias Cognitivas. 10(1), 19-28. http://dx.doi. org/10.5935/1808-5687.20140004

Macedo, D. M., Petersen, C. S., \& Koller, S. H. (2017). Desenvolvimento Cognitivo, socioemocional e físico na adolescência e as terapias cognitivas contemporâneas. In: Neufeld, C. B (Org). Terapia CognitivoComportamental para adolescentes: uma perspectiva transdiagnóstica e desenvolvimental. Porto Alegre: Artmed.
Matos, M. G., \& Sampaio, D. (2009). Jovens com Saúde: diálogo com uma geração. Lisboa: Texto Editores, LDA.

Matos, M. G., Simões, C., Tomé, G., Camacho, I., Ferreira, M., L., \& Aventura Social \& Saúde. (2011). Aventura social \& saúde: A saúde dos adolescentes portugueses: Relatório do estudo HBSC 2010 [Social adventure \& Health: The health of Portuguese adolescentes: Report of the 2010 HBSC study]. Cruz Quebrada: Faculdade de Motrocidade Humana.

Matos, G. M., Simões, C., Camacho, I., Reis, M., \& Equipa Aventura Social (2015). A saúde dos adolescentes portugueses em tempos de recessão - dados nacionais do estudo HBSC de 2014. Lisboa: Centro de Malária e Outras Doenças Tropicais /IHMT/UNL e FMH/ Universidade de Lisboa.

Matos, M. G. \& Equipa Aventura Social (2018). A saúde dos adolescentes após a recessão. Relatório do estudo Health Behaviour in School Aged Children (HBSC) em 2018. Disponível em www.aventurasocial.com

Neufeld, C. B. (2015). Terapia cognitivo comportamental em grupo para crianças $e$ adolescentes. Porto Alegre: Artmed.

Nogueira, J. M. (2017). Indicadores e comportamentos de saúde em adolescentes de 13 anos de Ribeirão Preto. Dissertação de Mestrado. Faculdade de Filosofia, Ciência e Letras de Ribeirão Preto da Universidade de São Paulo.

Ok, S., \& Aslan, S. (2010). The school bullying and perceived parental style in adolescents. Procedia-social and behavioral sciences, 5, 536-540. https://doi. org/10.1016/j.sbspro.2010.07.138

Reis, M., Figueira, I., Ramiro, L., \& Matos, M. G. (2012). Jovens e comportamentos de violência autodirigida. En M. G. Matos, G. Tomé \& Aventura Social, Promoção de competências e do capital social para um empreendendorismo com saúde na escola e na comunidade, pp. 259-276. Lisboa: Placebo Editora.

Rodrigues, M. (2015). Educação Emocional Positiva. Novo Hamburgo: Sinopsys Editora. Roberts, C., Freeman, J., Samdal, O., Schnohr, C. W., de Looze, M. E., Nic Gabhainn, 
S., Rasmussen, M. (2009). The Health Behaviour in School--aged Children (HBSC) study: Methodological developments and current tensions. International Journal of Public Health, 54(2), 140-150. http://dx.doi. org/10.1007/s00038-009-5405-9

Silva, A. K. L. S. (2013). Diversidade sexual e de gênero: a construção do sujeito social. Revista do NUFEN, 5(1), 12-25. Recuperado de http://pepsic.bvsalud.org/ scielo.php?script $=$ sci_arttext\&pid=\$21752 $5912013000100003 \&$ lng=pt\&tlng=pt.

Spence, S. H. (1998). A measure of anxiety symptoms among children. Behaviour Research and Therapy, 36, 545-566.

Tomé, G., \& Matos, M. G. (2006). Depressão, rendimento escolar e estratégias de coping em adolescentes. Revista Brasileira de Terapias Cognitivas, 2(1), 85-93. http:// dx.doi.org/10.5935/1808-5687.20060009

Tortorelli, M.F.P., Carreiro, L.R.R. \& Araújo, M.V. (2010). Correlações entre a percepção da violência familiar e o relato de violência na escola entre alunos da cidade de São Paulo. Psicologia Teoria e Prática, 12(1), 32-42. Recuperado de http://pepsic.bvsalud.org/ pdf/ptp/v12n1/v12n1a04.pdf

Warren, S. L., \& Sroufe, L. A. (2004). Developmental issues. In T. H. Ollendick \& J. S. March (Eds.), Phobic and anxiety disorders in children and adolescents: A clinician's guide to effective psychosocial and pharmacological interventions (pp. 92-115). New York, NY, US: Oxford University Press. 